\title{
A Specific Combination Scheme for Communication Modulation Recognition Based on the Bees Algorithm
}

\author{
Faquan YANG \\ School of Electronic and Information Engineering, \\ Foshan University \\ Guang Dong Foshan, China \\ Yafaquan.fosu@163.com
}

Zan LI

State Key Laboratory of Integrated Service Networks, Xidian University

Shaanxi Xian, China

Zanli@163.com

\author{
Yun FAN \\ School of Electronic and Information Engineering, \\ Foshan University \\ Guang Dong Foshan, China \\ Yunfan@163.com
}

\begin{abstract}
For the modulation recognition of the wireless communication signal, when extracting Eigenvalues, it is necessary to improve modulation recognition rate in order to achieve the optimized effect. In this article, combination eigenvalue module of the signal is extracted by applying bee colony algorithm and automatic recognition of the communication signal is achieved through the classifier which has multi-layer sensor neural network. The simulation results show that the proposed algorithm in this paper can result the communication signal modulation recognition rate is higher than the corresponding rate in using the conventional method under the conditions that the number of neurons is only 20 in the hidden layers, and the system is easy to realize, it has wide application prospect in signal recognition.
\end{abstract}

Keywords-wireless communication signal; bee colony algorithm; combination eigenvalue module; multi-layer perceptron neural network; modulation recognition

\section{INTRODUCTION}

Automatic recognition of the communication signal is the basic of the software-defined ratio, cognitive ratio, spectrum sensing and other fields. It is widely used in military use and civilian use[1-2].

In the automatic recognition of the communication signal, the extract of the eigenvalues is significant. The current common way of extracting eigenvalues mainly include extracting eigenvalues in the frequency domain and time-domain respectively, analyzing high-order original moment, high-order cumulant, spectral correlation, cyclic spectral correlation, power spectrum, constellation diagram, kurtosis of the signal and other parameters; wavelet transform etc[3-5]. In order to improve recognition rate, simultaneous extract multiple eigenvalues and achieved interclass and outside the class recognition by trying different ways, which are introduced in the recent related journal in both domestic and aboard [6-7] and the effect of the work is also impressive. However, how to constantly innovative based on the current ways and improve modulation recognition rate are still challenging research topic.

Therefore, at the same time of extracting multiple eigenvalues, in order to obtain the optimization effect, bees algorithm (BA) was introduced in this article and these time-domain eigenvalues, frequency domain eigenvalues, high-order original moment, high-order cumulant and their various numbers of combination as combination eigenvalue module are extracted, on this foundation through neural network classifier achieved signal automatic recognition and achieved good results.

\section{COMBINATION EIGENVALUE MODULE}

The combination eigenvalue module extraction by BA includes $\gamma_{\max }, \sigma_{o f}, \sigma_{o p}$, high-order original moment, high-order cumulant and their various numbers of combination. When intercepting the signal, $\gamma_{\max }$ is the maximum of the center the instantaneous amplitude power spectrum density, $\sigma_{\text {of }}$ is the standard deviation of the normalized instantaneous frequency absolute value and $\sigma_{o p}$ is the standard deviation of the normalized instantaneous phase nonlinear component absolute value in the period of not weak signals.

\section{A. High-order original moment}

A random variable $\boldsymbol{s}$ in the $i$ order original moment can be defined as:

$$
\mu_{i}=\int_{-\infty}^{\infty}(s-m)^{i} f(s) \mathrm{d} \boldsymbol{s}
$$

$m$ is mean value of random variable so the $i$ order original moment when the length is $N$ can be presented as: 


$$
\mu_{i}=\sum_{k=1}^{N}\left(\boldsymbol{s}_{k}-m\right)^{i} f\left(\boldsymbol{s}_{k}\right) \mathrm{d} \boldsymbol{s}
$$

If mean value of signal is 0 , the $i$ order original moment when the length is $N$ can be presented as:

$$
\mu_{i}=\sum_{k=1}^{N} \boldsymbol{s}_{k}^{i} f\left(\boldsymbol{s}_{k}\right) \mathrm{d} \boldsymbol{s}
$$

The autocorrelative original moment of random variable $\boldsymbol{s}$ is defined as:

$$
M_{p q}=E\left[\boldsymbol{s}^{p-q}\left(\boldsymbol{s}^{*}\right)^{q}\right]
$$

$p$ is the order of original moment, $\boldsymbol{s}^{*}$ is the complex conjugate of random variable $s$. Set 0 mean value baseband sequence as: $\boldsymbol{s}_{k}=a_{k}+\mathrm{j} b_{k}$.According to the definition of autocorrelative original moment with different orders, the original moment with different orders can be calculated as:

$$
\begin{aligned}
& M_{84}=E\left[s^{4}\left(s^{*}\right)^{4}\right]=a^{8}+10 a^{6} b^{2}+ \\
& 16 a^{4} b^{4}-16 a^{2} b^{6}+21 a^{4} b^{4}-12 a^{2} b^{2}+b^{8}
\end{aligned}
$$

Among (4), these higher order origin moment eigenvalues of 2PSK,16QAM,64QAM which are applied in simulation experiment are shown in table 1

TABLE I. EIGENVALUES OF HIGHER ORDER ORIGIN MOMENT

$\begin{array}{cccc}\text { eigenvalues } & M_{41} & M_{61} & M_{84} \\ \text { 2PSK } & 1 & 1 & 1 \\ \text { 16QAM } & 0 & -1.32 & 3.13 \\ \text { 64QAM } & 0 & -1.3 & 3.9\end{array}$

\section{B. High-order cumulant}

The random variable $s$ under condition that the mean value is set as 0 , the eigenfunction is $\hat{f}(t)$, expand it as the Taylor Series and is represented as:

$$
\log \hat{f}(t)=k_{1}(j t)+\ldots .+\frac{k_{r}(j t)^{r}}{r !}+\ldots .
$$

Among (5), $K_{r}$ is called the cumulant of the random variable $\boldsymbol{S}$. The formula under the $p$ order cumulant is similar to the formula under the $p$ order original moment, and they are showed as:

$$
C_{p q}=\operatorname{Cum}\left[s \ldots . . s, s^{*}, \ldots s^{*}\right]
$$

Among (6), $s \ldots s$ has $p-q$ number, $s^{*} \ldots . . s^{*}$ has $q$ number. For example:

$$
\boldsymbol{C}_{84}=C u m=\left(s, s, s, s, s^{*}, s^{*}, s^{*}, s^{*}\right)
$$

The cumulant under the $n$ order is the function of the original moment whose order accumulates to $n$. According to the definition of accumulates, this original moment can be showed as:

$$
\begin{gathered}
M\left[\boldsymbol{s}_{1}, \cdots, \boldsymbol{s}_{n}\right]=\sum_{\forall p} \operatorname{Cum}\left[\left(\boldsymbol{s}_{j}\right)_{j e v_{1}} \cdots\right. \\
\operatorname{Cum}\left[\left(\boldsymbol{s}_{j}\right)_{j \in v_{q}}\right.
\end{gathered}
$$

According to the definition of the original moment, the cumulant can also be showed as:

$$
\begin{aligned}
\operatorname{Cum}\left[s_{1}, \cdots, s_{n}\right]= & \sum_{\forall v}(-1)^{q-1}(q-1) ! \times \\
& E\left[\prod_{j \in v_{1}} s_{j}\right] \cdots E\left[\prod_{j \in v_{p}} s_{j}\right]
\end{aligned}
$$

Among (8), each component $v=\left(v_{1}, \cdots, v_{q}\right)$ related to the index $(1,2,,,,, n)$ is accumulated. If the high order cumulant under the order 2 , order 4 , order 6 ,order 8 can be calculated respectively, 14 types of high-order cumulant including $\boldsymbol{C}_{20}, \boldsymbol{C}_{21}, \boldsymbol{C}_{40}, \boldsymbol{C}_{41}, \boldsymbol{C}_{42}, \boldsymbol{C}_{60}, \boldsymbol{C}_{61}, \boldsymbol{C}_{62}$, $\boldsymbol{C}_{63}, \boldsymbol{C}_{80}, \boldsymbol{C}_{81}, \boldsymbol{C}_{82}, \boldsymbol{C}_{83}$, and $\boldsymbol{C}_{84}$ are regarded as the characteristic of the signal [12-13].Three input signals higher order cumulant eigenvalues of 2PSK,16QAM,64QAM which are applied in simulation experiment are shown in table 2

TABLE II. EIGENVALUES OF HIGHER ORDER CUMULANT

$\begin{array}{cccc}\text { eigenvalues } & C_{61} & C_{80} & C_{84} \\ \text { 2PSK } & 16 & -244 & -244 \\ \text { 16QAM } & 2.08 & -13.99 & 17.38 \\ \text { 64QAM } & 1.797 & -11.5 & 0\end{array}$

\section{THE EXTRACTION OF COMBINATION EIGENVALUE MODULE THROUGH BA}

The principle of extracting the combination eigenvalue through BA is based on the model of bee colony's nature secret behavior. The optimal solution can be found through the implementation of global search strategy and the algorithm of the strategy of the local neighborhood search The principle is:

The first process is random initialization. $n$ worker bees reconnoiter and the worker bees will search continuously for the whole solution space in this algorithm. Therefore, the updated formula is:

$$
\begin{array}{r}
x_{i}=\min +\operatorname{rand}(0,1)(\max -\min ) \\
i \in(m+1, m+2, \cdots, n)
\end{array}
$$

Among (9), min is the next value of solution space and max is the previous value of the solution space.

In the iterative process of finding the optimal solution, $m$ better worker bees are firstly selected. After the selection, these better worker bees in the flowers should be reinforced respectively. The worker bees reinforced will be generated by the following formula:

$$
\begin{aligned}
v= & \left(x_{i}-n g h\right)+2 \times n g h \times \operatorname{rand}(0,1) \\
& i \in(m+1, m+2, \cdots, n)
\end{aligned}
$$

Among (10), $n g h$ is the radius of flowers and presents the neighbourhood search area.

Selecting the best worker bees $v_{\text {best }}$ in these worker bees reinforced. If $v_{\text {best }}$ is better than current worker bees $x_{j}, x_{j}$ will be replaced by $v_{\text {best }}$.

After each iteration, $m$ most optimal worker bees are retained and other $n-m$ worse worker bees are updated iterated based on (9). Only when a satisfactory 
combination eigenvalue module is obtained (also called the extraction of the satisfactory combination eigenvalue module), the optimization process finish. Otherwise, return, continuously iterated, and update so as to find the optimal combination eigenvalue module[14].

For three kinds of modulating signal of 2PSK,16QAM,64QAM which are applied in simulation experiment, corresponding parameter values of combination eigenvalue module extraction by $\mathrm{BA}$ are shown in table 3

TABLE III. CORRESPONDING PARAMETER VALUES OF COMBINATION EIGENVALUE MODULE EXTRACTION BY BA ALGORITHM

\begin{tabular}{|c|c|c|c|c|c|}
\hline $\mathrm{n}$ & $\mathrm{m}$ & $\mathrm{e}$ & $\mathrm{ngh}$ & $\min$ & $\max$ \\
\hline 30 & 8 & 2 & 0.1 & 4 & 15 \\
\hline
\end{tabular}

\section{MLP NEURAL NETWORK CLASSIFIER BASED ON BP ALGORITHM}

\section{A. MLP neural Network Classifier}

There are different types of MLP neural network classifier. Take feedforward for example, it is composed of input layer, hidden layer and out layer. In the input layer, each input in the input layer is fed to the hidden layer. Then output of each cell in the hidden layer is connected to each neural cell of its next layer called output layer. As a feedforward network, it generally can have any number of hidden layers[15].

But when dealing with most of the problem, a hidden layer is usually enough. In addition, the number of the cellular network can also be completely arbitrary and each layer can actually have any number of nerve cells, which is completely based on the complexity of the problem to be solved. But if more nerve cells is, lower the network speed is. Therefore, the scale of the network is always required to keep as small as possible[16].

\section{B. BP Algorithm used in classifier}

Error back-propagation algorithm(BP algorithm), as the BP algorithm has significant advantages such as simple, easy, strong parallelism and needs small amount of calculation, it is one of the most mature training algorithm which is applied in the current MLP neural network training. The basic idea of BP algorithm is the learning process consists of signal forward propagation and error back propagation. Meanwhile, through the output error back propagation, the error can be divided into all units in each layer so as to obtain the error signal of each unit and fix the weights of each unit. Then the rule of the weight change in the BP algorithm is:

$$
x_{i j}(t+1)=x_{i j}(t)+a_{i j} g_{i j}(t)
$$

Among (11), $x_{i j}(t)$ represents the weight of cells from $i$ to $j$ at time $t . x_{i j}(t+1)$ represents the weight of cells from $i$ to $j$ at time $t+1 . g_{i j}(t)$ represents the weight change gradient of cells from $i$ to $j$ at time $t . a_{i j}$ represents the learning rate of cells from $i$ to $j$.

\section{THE MODULATING RECOGNITION SIMULATION OF} THE SYSTEM AND THE ANALYSE OF ITS PERFORMANCE

The system adopts BA algorithm. Different number of combination eigenvalue module is extracted through the most optimized solution and then they are sent to the classifier as afferent characteristic parameter vector of MLP neural network. Then MLP classifier is executed learning and training through BP algorithm training, which achieves the modulating recognition of three modulating signal (2PSK, 16QAM and 64QAM).

The condition of simulation parameters are using random sequence as a modulation signal while keeping symbol rate $R_{S}=12 \mathrm{~kb} / \mathrm{s}$ and the carrier frequency of 2PSK,16QAM,64QAM for modulation identification signal is $200 \mathrm{kHz}$; three-layer feed forward network is applied in neural network; the number of hidden layer neurons is 20 and the transfer function among input layer, hidden layer and output layer is hyperbolic tangent sshaped function because there are three kinds of types modulation signal to be identified;. Mean square error is $10^{-6}$, white gaussian noise channel, SNR is $-4 \mathrm{~dB} \sim 12 \mathrm{~dB}$, training sample for 2000 .

Different number of combination eigenvalue module is extracted by applying BA so as to conducting the most optimized process. Three combination eigenvalue module is respectively $\left(\gamma_{\max }, \sigma_{o f}, \sigma_{o p}, M_{41}, C_{61}\right)$ (five eigenvalues), $\left(\gamma_{\max }, \sigma_{o f}, \sigma_{o p}, M_{41}, M_{61}, \boldsymbol{C}_{61}, \boldsymbol{C}_{80}\right)$ (seven eigenvalues $)$, and $\left(\gamma_{\max }, \sigma_{o f}, \sigma_{o p}, M_{41}, M_{61}, M_{84}, \boldsymbol{C}_{61}\right.$ $\boldsymbol{C}_{84}, \boldsymbol{C}_{80}$ )(nine eigenvalues). After MLP classifier is conducted training and learning through the BP algorithm, the modulation recognition of the afferent 2PSK, 16QAM and 64QAM is conducted and the result of simulation is shown in the Fig.1-3.

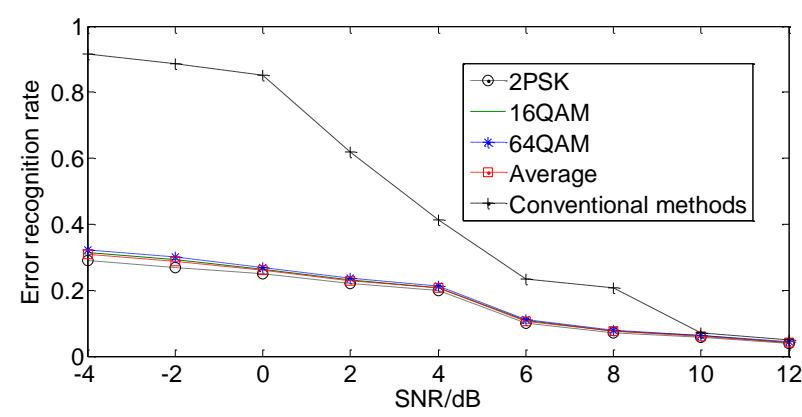

Figure 1. The result of simulation (five eigenvalue)

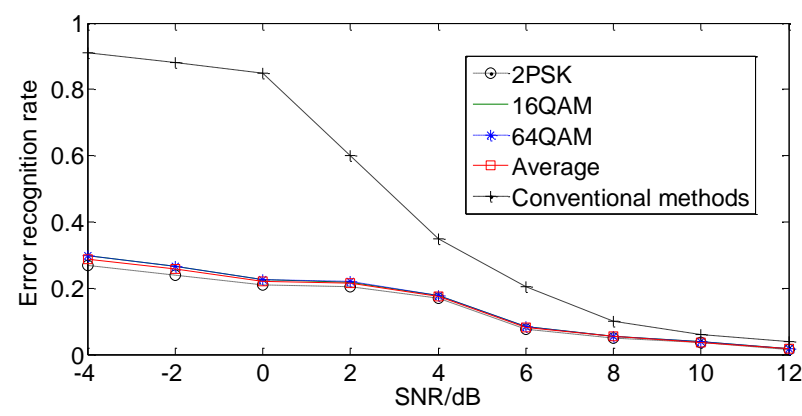

Figure 2. The result of simulation (seven eigenvalue) 


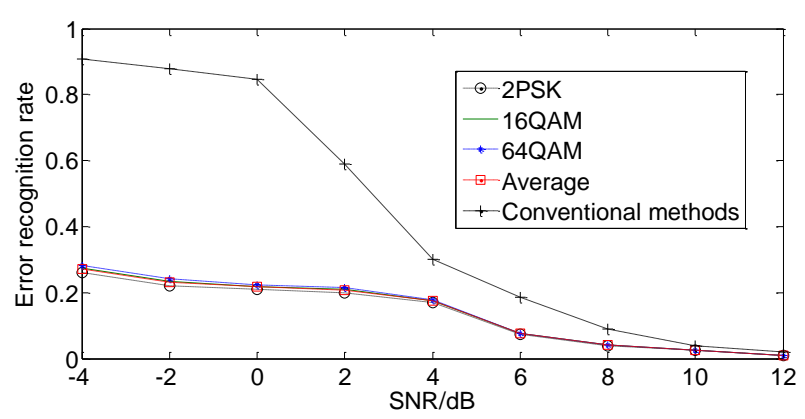

Figure 3. The result of simulation (nine eigenvalue)

The conventional method shown in Fig .1-3 means the extracted combination eigenvalue module of the signals which are to be recognized is sent to the MLP neural network classifier which is based on BP algorithm and is trained directly, so as to achieve modulation recognition of three kinds of the input signals. From Fig .1-3, comparing modulation recognition of three modulated signal including 2PSK, 16QAM, AND 64QAM by the conventional method with applying BA mentioned in this paper, the results show that the average recognition is much smaller, especially under the condition that the signal-to-noise ratio is low. In addition, by comparing with the Fig .1-3, it is obvious to find out the more eigenvalues which is included in the extracted combination eigenvalue module, the lower error probability in recognizing, especially under low signal-to-noise ratio (less than $0 \mathrm{~dB}$ ). When the number of the eigenvalues in the combination eigenvalue module can reach a certain extent, such as more than 7 , the system modulation recognition rate begin increasing slightly. Therefore, it can be predicted that the recognition rate will no longer improve when the number of combination of characteristic values reach a certain degree because the optimization of the extracted combination eigenvalue module through $\mathrm{BA}$ has reached the limiting condition.

\section{CONCLUSION}

The extraction based on the BA in this paper is combination eigenvalue module which is composed of $\gamma_{\max }, \sigma_{o f}, \sigma_{o p}$ and different number of higher-order cumulants,origin moment. Then based on it, BP algorithm is used to train MLP neural network classifier to achieve the modulation recognition of communication signal while being compared with the conventional methods. The simulation results show that the proposed algorithm in this paper can result the communication signal modulation recognition rate is higher than the corresponding rate in using the conventional method. Three kinds of wireless communication signals modulation recognition rates on average will be higher than $90 \%$ under the condition that the number of the neurons is only 20 in the hidden layers and the signal to noise ratio is $6 \mathrm{~dB}$.

\section{ACKNOWLEDGMENT}

I wish to thank my classmates and friends in our laboratory such as Dr. Zhongxian PAN, Dr. Benjian Hao et al. This work was supported in part by a grant from my work affiliation: school of electronic and information engineering, Foshan University. In addition, the paper support by the National Natural Science Foundation of China (61072070 and 61301179), the Doctorial Programs Foundation of the Ministry of Education (20110203110011), the Fundamental Research Funds of the Ministry of Education (72124338), the Small and Midsized Enterprise of Science and Technology Innovation Fund (14C26214402603), the Science and Technology Plan Projects of Guangdong Province (2011B010200030, 2011B010100038).

\section{REFERENCES}

[1] Z.M.Yu,"The research of signal recognition technologies in wireless communication,"Harbin Engineering University, Dec. 2010 .

[2] F.Q.Yang, Z.L, Z.L.Luo, "A New Method of Modulation Recognition of Block Orthogonal Amplitude Modulation,” Journal of Communications, Sep.2014, 9(9): 693-698.

[3] Y.E. Wang, T. Q.Zhang et al,"Recognition of OFDM signals based on cyclic autocorrelation," Video Engineering,2012,68(5):44-48.

[4] X.J.Wei, H. Xie, "Digital modulation recognition algorithm based on support vector machine," Electronic Design Engineering, 2011,35(6):89-91.

[5] E.Nguifo, N.Tsopze, G.Tindo, "Multiclass concept lattice-Based artificial neural network," Studies in Computational Intelligence, 2013, 25(8):103-121.

[6] Z.N.Xu,W.L.Bai,“A novel blind recognition algorithm for modulated M-QAM signals,"Communications and Mobile Computing of the 2011 International Conference, Dec.2011: 461465.

[7] F.Q.Yang,Z.L,Z.L.Luo, "Research of VHF Band Signal Modulation Classification and Recognition Methods Based on Algorithm of First-Order Cyclic moment" Telecommunications Science, Feb.2014, 30(2):76-81.

[8] Z C. Z.hang,G.Q.Zheng,“ Overview of research on bee colony algorithms," Application Research Computers,2011,28(9):1-5.

[9] C.Glavan, S.Holbn, "A model for determining the number of negative examples used in training a MLP,"Innovations in Computing Sciences and Software Engineering,2010, 55(2):537542 .

[10] A. E.Sherme, H. A.Zimi, "Blind signal- type classification using a novel robust feature subset selection method and neural network classifier,"Annals of Telecommunications, 2010, 65(5): 625-633.

[11] Y. F.Cabrera, R.Perez, Y. C.Mota,"Improving the MLP learning by using a method to calculate the initial weights of the network based on the quality of similarity measure," Advances in Soft Computing, 2011, 70(5): 351-362.

[12] L. M.Ai, C.Guo, "Tire tread pattern recognition based on composite feature extraction and hierarchical support vector machine," Computer Engineering and Applications, 2012, 48(6):14-17.

[13] X. L.Zhang, H. Q.Li, "Modulation recognition based on joint characteristics in spectrum domain,"Wireless Communication Technology,2010,35(5): 59-61.

[14] F.Q.Yang, Z.L, Z.L.Luo, "Method of Neural Network Modulation Recognition Based on Clustering and Polak-Ribiere Algorithm," Journal of Systems Engineering and Electronics, Nov.2014, 25(5): 742-747.

[15] J.Yu, Y.Chen, "Digital modulation recognition method based on BP neural network,'Transducer and Microsystem Technologies, 2012,31(5): 16-19.

[16] Y.Zhou, A. F.Zhu, "Sample data selection method for neural network classifiers," Journal of Huazhong University of Science and Technolo- gy(Nature Science Edition), 2012,40(6): 23-26. 